Review

\title{
Molecular Mechanisms and Clinical Impact of Acquired and Intrinsic Fosfomycin Resistance
}

\author{
Alfredo Castañeda-García ${ }^{1, *}$, Jesús Blázquez ${ }^{2, *}$ and Alexandro Rodríguez-Rojas ${ }^{3 \% *}$ \\ 1 Genome Damage and Stability Centre, University of Sussex, Falmer, Science Park Road, Brighton, \\ East Sussex BN1 9RQ, UK \\ 2 Centro Nacional de Biotecnología (CSIC), Departamento de Biotecnología Microbiana, Calle \\ Darwin 3, 28049 Madrid, Spain \\ 3 Institute for Biology, Freie Universität Berlin, Königin-Luise-Str. 1-3, 14195 Berlin, Germany \\ * Authors to whom correspondence should be addressed; E-Mails: A.Castaneda-Garcia@sussex.ac.uk \\ (A.C.-G.); blazquez@cnb.csic.es (J.B.); a.rojas@fu-berlin.de (A.R.-R.).
}

Received: 11 March 2013; in revised form: 5 April 2013 / Accepted: 8 April 2013 /

Published: 16 April 2013

\begin{abstract}
Bacterial infections caused by antibiotic-resistant isolates have become a major health problem in recent years, since they are very difficult to treat, leading to an increase in morbidity and mortality. Fosfomycin is a broad-spectrum bactericidal antibiotic that inhibits cell wall biosynthesis in both Gram-negative and Gram-positive bacteria. This antibiotic has a unique mechanism of action and inhibits the initial step in peptidoglycan biosynthesis by blocking the enzyme, MurA. Fosfomycin has been used successfully for the treatment of urinary tract infections for a long time, but the increased emergence of antibiotic resistance has made fosfomycin a suitable candidate for the treatment of infections caused by multidrug-resistant pathogens, especially in combination with other therapeutic partners. The acquisition of fosfomycin resistance could threaten the reintroduction of this antibiotic for the treatment of bacterial infection. Here, we analyse the mechanism of action and molecular mechanisms for the development of fosfomycin resistance, including the modification of the antibiotic target, reduced antibiotic uptake and antibiotic inactivation. In addition, we describe the role of each pathway in clinical isolates.
\end{abstract}

Keywords: fosfomycin resistance; molecular mechanisms 


\section{Introduction}

Infectious diseases are the second-major cause of death worldwide and the third-leading cause of death in economically advanced countries [1]. Antibiotic-resistant strains of pathogenic bacteria are increasingly prevalent and represent a priority health problem [2]; hence, the problem of antibiotic resistance needs an urgent response. Developing a new antibiotic can take years and millions of dollars. Therefore, in the meantime, the rational use or retrieval of old antibiotics, like polymyxins, fusidic acid, co-trimoxazole, aminoglycosides, chloramphenicol and fosfomycin, may be a short-term solution [3].

Here, we focus our analysis on fosfomycin (also termed phosphomycin and phosphonomycin), a natural antibiotic compound produced by several Streptomyces and Pseudomonas species, exerting a powerful bactericidal activity against a wide range of Gram-negative and Gram-positive bacteria [4]. Fosfomycin is a phosphonic acid derivative containing an epoxide and a propyl group [(2R,3S-3-methyloxiran-2-yl) phosphonic acid] with a unique chemical structure (Figure 1). This molecule, with a very low molecular weight, is within a class of its own and is unrelated to any other antibiotic family, in addition to having an exclusive target, the initial step in peptidoglycan biosynthesis [5].

Figure 1. Chemical structure of fosfomycin [(2R,3S-3-methyloxiran-2-yl) phosphonic acid].

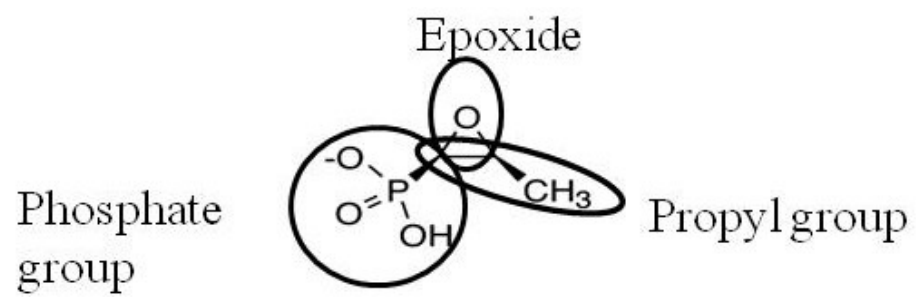

\section{Activity}

Fosfomycin is mainly used for the treatment of uncomplicated urinary tract infections (UTIs) [6], and various formulations are available. The form of the medication for intravenous use is fosfomycin disodium salt. For oral use, the antibiotic is combined in calcium salt or formulated with tromethamine. Fosfomycin tromethamine is primarily administered in a single dose, reaching a very high antibiotic concentration that is able to successfully kill the most common urinary pathogens [7]. It has also been used in combination with other antibiotics in the treatment of patients suffering serious infections, systemic infections with sepsis or nosocomial infections [8].

Fosfomycin shows a powerful bactericidal activity against enteric Gram-negative bacteria, such as Escherichia coli, although some of them have decreased susceptibility, as Klebsiella pneumoniae and Enterobacter cloacae. Fosfomycin is also very effective against Gram-positive cocci, like Staphylococcus aureus, Streptococcus pneumoniae, Enterococcus faecalis and Enterococcus faecium [9].

The analysis of its activity against nine commonly encountered bacteria associated with urinary tract infection has revealed a high susceptibility in E. coli isolates and most $K$. pneumoniae, E. cloacae and $S$. aureus strains [10]. In addition, E. faecalis and E. faecium isolates were also quite susceptible to fosfomycin, yet with higher MIC values. However, Acinetobacter baumannii isolates were resistant to fosfomycin, while P. aeruginosa and Stenotrophomonas maltophilia showed moderate susceptibility [10]. 
Fosfomycin has been successfully evaluated as a treatment option for infections caused by multiple drug resistant (MDR) Gram-negative and Gram-positive bacteria [11,12]. For example, a survey of clinical MDR Enterobacteriaceae isolates, including producers of extended-spectrum $\beta$-lactamases (ESBL), showed that $>90 \%$ of $E$. coli and $>80 \%$ of $K$. pneumoniae isolates were susceptible to fosfomycin [13].

\section{Mechanism of Action}

Fosfomycin is a bactericidal antibiotic that inhibits the initial step in the biosynthesis of peptidoglycan in prokaryotes [5]. Peptidoglycan is assembled from a building block composed of $\mathrm{N}$-acetylglucosamine (GlcNAc) and $\mathrm{N}$-acetylmuramic acid with an attached pentapeptide. Fosfomycin acts as a phosphoenolpyruvate (PEP) analogue and binds MurA (UDP-GlcNAc enolpyruvyl transferase), an essential enzyme for peptidoglycan biosynthesis [14], preventing the formation of UDP-GlcNac-3O-enolpyruvate from UDP-GlcNAc and PEP during the first step in peptidoglycan biosynthesis, leading to bacterial cell lysis and death [5] (Figure 2). The antibiotic can enter into the active site of MurA and inhibits this enzyme by covalently binding via a thioether bond formation with a key residue in the active site, Cys115 [15,16]. The crystal structure of E. coli MurA complexed with UDP-GlcNAc and fosfomycin has revealed that the Cys115-bound molecule is tightly packed between the enzyme and the substrate, forming strong electrostatic interactions between three conserved positively charged residues of MurA (Lys22, Arg120 and Arg397) and the phosphonate group of the antibiotic [16].

\subsection{Mechanisms of Fosfomycin Resistance}

There are different mechanisms leading to fosfomycin resistance:

(i) Reduced permeability to fosfomycin. Since the discovery of fosfomycin, it was established that the main mechanism for the acquisition of antibiotic resistance was an impaired fosfomycin transport, due to mutation of any of the target genes encoding the antibiotic permeases. In E. coli, two main nutrient transport systems are responsible for fosfomycin uptake, the glycerol-3-phosphate transporter (GlpT) and a hexose phosphate transporter, the glucose-6-phosphate transporter (UhpT) [5]. The expression of the GlpT and UhpT transporters is induced by their substrates, glycerol-3-P and glucose-6-P, respectively, and requires the presence of cAMP-CRP (Figure 3). Mutations in any of the structural genes of those pathways produce a decrease in antibiotic uptake, conferring different levels of fosfomycin resistance [5,17,18].

Strains defective in fosfomycin uptake are not able to grow using some substrates as the sole carbon source, glycerol-3-P in GlpT-deficient strains or glucose-6-P (and other hexose phosphates) in Uhp-deficient strains. Mutants affected in both systems are often unable to grow using multiple carbohydrates. In fact, it has been observed that the addition of glucose-6-P induces fosfomycin sensitivity in resistant GlpT-deficient strains, due to the induction of UhpT synthesis [5]. Therefore, the measurement of MIC to fosfomycin in E. coli is performed using media with and without glucose-6-P [19]. However, the addition of glucose-6-P recommended by the CLSI manual provides a more reliable MIC result, due to its activity as inducer of fosfomycin transport. 
Figure 2. Although transporters are usually very selective, the chemical structure of fosfomycin mimics both glycerol-3-P (G3P) and glucose-6-P (G6P), which are transported under normal conditions. MurA catalyses the formation of UDP-GlcNac-3-O-enolpyruvate, a peptidoglycan precursor, from UDP-GlcNAc and PEP during the first step of peptidoglycan biosynthesis, allowing cell growth (A). In contrast, when fosfomycin (F) is present, it is transported inside the cell by GlpT and UhpT, blocking the UDP-GlcNac-3-O-enolpyruvate synthesis by mimicking the original substrate of MurA, PEP, avoiding cell wall synthesis and leading to cell death (B). For simplicity, only peptidoglycan and the inner membrane are shown.

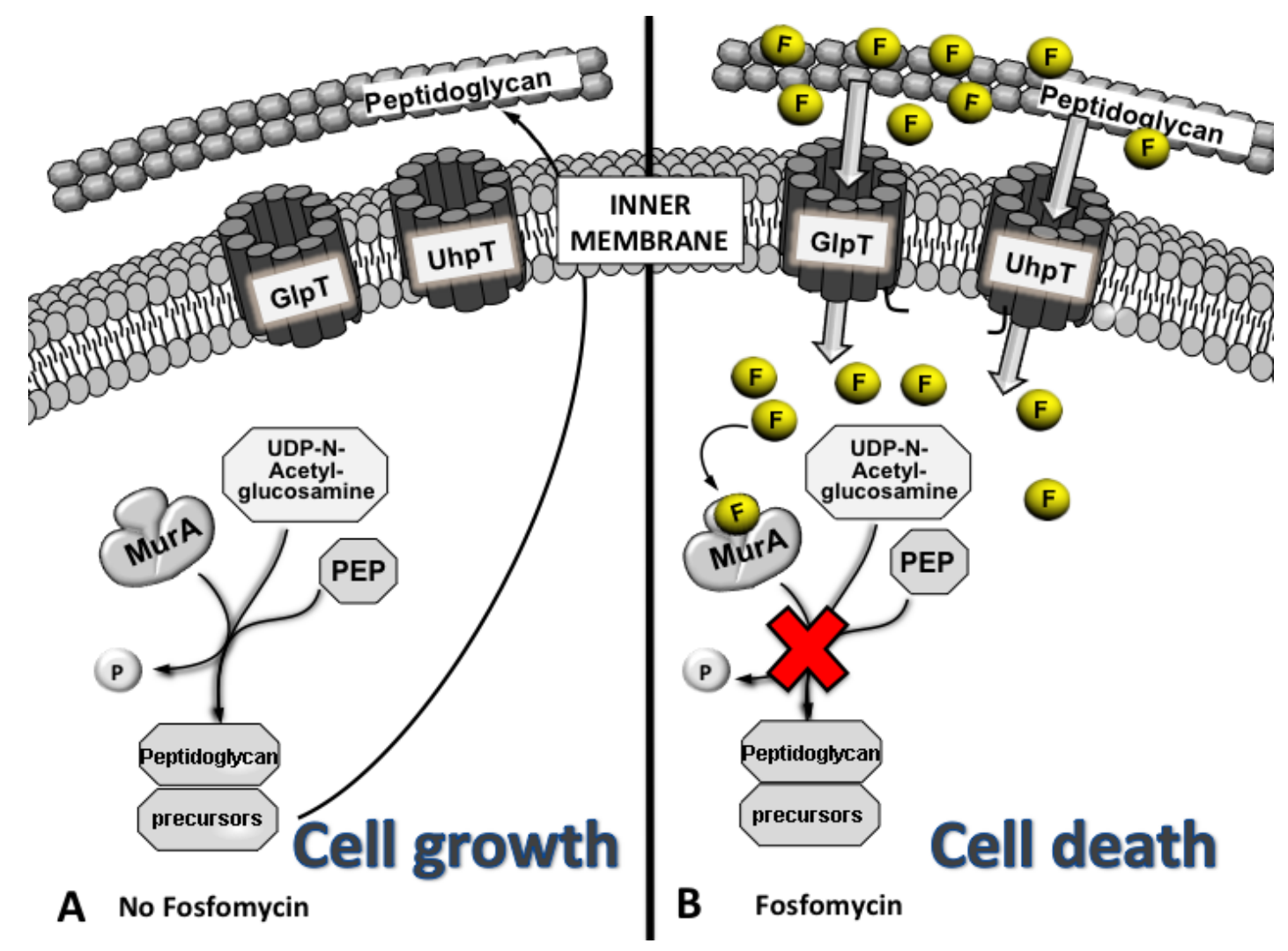

While fosfomycin uptake depends on GlpT and UhpT in E. coli, it has been reported that fosfomycin can only enter into the cells via GlpT in P. aeruginosa, due to the absence of UhpT permease. As a result, $g l p T$ is the only target gene whose inactivation confers antibiotic resistance in $P$. aeruginosa [20]. All the fosfomycin-resistant mutants generated in $P$. aeruginosa by spontaneous mutations are affected in $g l p T$ [20]. However, these mutations appear to be cost-free, probably because this species cannot use glycerol-3-P as a sole carbon source, even in $g l p T^{+}$wild-type strains [21].

Fosfomycin uptake is essential for antibiotic activity, and intrinsic resistance to the antibiotic in some pathogenic species is caused by the lack of transport. A paradigmatic example is Listeria monocytogenes. This species is unable to uptake the antibiotic in vitro and, consequently, is resistant to fosfomycin. Nevertheless, a central virulence regulator, PrfA, induces in vivo the virulence factor Hpt, a glucose-6-P permease that also mediates the uptake of fosfomycin, conferring antibiotic susceptibility during infection [22,23]. 
Figure 3. Regulation of GlpT and UhpT. In E. coli and several Enterobacteria, the expression of $g l p T$ and $u h p T$ requires the presence of the cAMP, which together with the receptor protein complex (CRP) forms the cAMP receptor protein complex (cAMP-CRP). This complex binds to the specific promoter sites of both genes, $g l p T$ and $u h p T$, and promotes their expression. Both transporters experience additional regulation. On the one hand, $g l p T$ gene expression is also controlled by the repressor, GlpR, which becomes inactive when it is bound to glycerol-3-P (G3P), and on the other hand, of $u h p T$; high-level expression also requires the regulatory genes, $u h p A, u h p B$ and $u h p C$, which sense and transduce signals by phosphorylation when hexose phosphates are detected, thereby positively regulating the transcription of the gene.

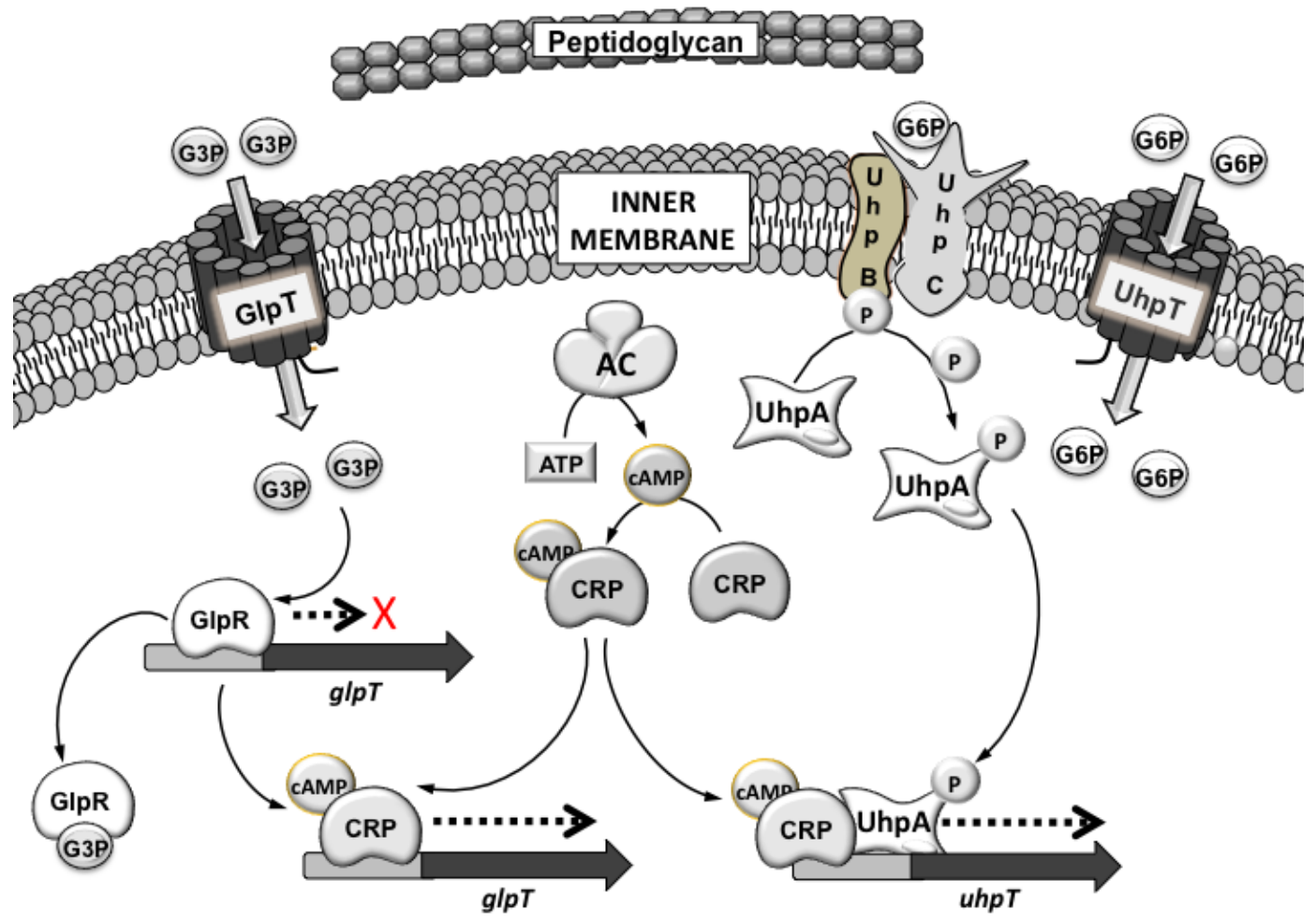

GlpT system. GlpT is a glycerol-3-phospate permease, a protein belonging to the organophosphate phosphate antiporter (OPA) family of the major facilitator superfamily (MFS). GlpT transporters are present in various bacterial species with a high degree of sequence conservation, and homologues are widely distributed in all phyla [24].

The structure of $E$. coli GlpT reveals two domains connected by a long central loop, with a substrate translocation pore located between the two domains opened to the cytoplasm $[25,26]$. As an integral inner membrane component, the GlpT protein contains 12 highly conserved transmembrane $\alpha$-helices typical of all glycerol-3-P permeases [25,26]. GlpT catalyses an exchange of external glycerol-3-P for internal $\mathrm{Pi}$, acting as a secondary active transporter for glycerol-3-P uptake [27].

This permease also provides an entrance mechanism for fosfomycin, owing to this antibiotic mimicking glycerol-3-P. The acquisition of mutations affecting GlpT confers fosfomycin resistance, decreasing the antibiotic uptake into the bacterial cells [5,28,29]. The interaction between GlpT and fosfomycin has recently been characterized in proteoliposomes, showing that fosfomycin competes for the substrate-binding site of the permease and is transported by the protein in vitro [30]. 
The expression of GlpT in E. coli is induced by the presence of glycerol-3-P. The repressor, GlpR, blocks $g l p T$ transcription by binding to the operators near the $g l p T$ promoter. The interaction of GlpR with glycerol-3-P reduces its affinity for the glpT operator and activates GlpT synthesis [31-33] (Figure 3). Inactivation of GlpR leads to a constitutive expression of $g l p T$.

UhpT system. UhpT is a hexose phosphate transporter responsible for the accumulation of glucose-6-P and the uptake of fosfomycin into the bacterial cells. Fosfomycin-resistant strains defective in growth with hexose phosphates as a carbon source, such as glucose-6-P, carry mutations in the $u h p$ genes [34].

UhpT is also a member of the Major Facilitator Superfamily that exchanges a cytoplasmic phosphate Pi for a hexose phosphate [35]. UhpT transporters show an extensive amino acid sequence homology with glycerol-3-P transporters, GlpT [36]. In a similar way as GlpT, UhpT is a monomer, with twelve transmembrane alpha-helical segments [37,38].

In addition, the UhpT transport system is controlled by several regulatory components, including $u h p A$, $u h p B$ and $u h p C$, whose products are necessary for high-level expression of the UhpT transporter (Figure 3). Inactivation of any of these regulatory genes also leads to fosfomycin resistance, due to an inhibited or reduced expression of the UhpT transporter, thereby preventing fosfomycin uptake into the cells [34].

The integral membrane component UhpC detects the extracellular signal, glucose-6-P and activates UhpB [39]. UhpB is a sensor histidine kinase in a two-component regulatory system with UhpA based on His-to-Asp phosphoryl transfer. UhpA, a DNA-binding response regulator, binds to two adjacent regions, an upstream strong binding site and a downstream weak binding site, in the $u h p T$ promoter. Phosphorylation of UhpA stimulates DNA binding, hence promoting $u h p T$ transcription [40-42] (Figure 3).

Regulation: levels of cyclic adenosine monophosphate (cAMP). High levels of cAMP are required for the full expression of the fosfomycin transporters, GlpT and UhpT, in Enterobacteria. cAMP synthesis depends on the activity of adenyl cyclase CyaA. cAMP levels are also regulated by the phosphotransferase enzyme, PtsI, a component of the PEP sugar phosphotransferase transport system. Mutations in cyaA or ptsI cause a decrease in the intracellular cAMP levels and, consequently, a reduced synthesis of both fosfomycin transporters, leading to a diminished antibiotic uptake [43-46].

Inactivation of the cAMP receptor protein CRP impairs the expression of both transporter systems, reducing the susceptibility to fosfomycin [43]. cAMP-CRP recognizes several binding sites upstream of the $g l p T Q$ operon in a DNA stretch, controlled negatively by GlpR and positively by cAMP-CRP [47]. This global regulator also binds to the E. coli uhpT promoter at a single site upstream of the UhpA-binding sites (Figure 3). Transcription of the $u h p T$ gene requires the response regulator UhpA and is stimulated by the global regulator protein, cAMP-CRP [48]. cAMP-CRP stabilizes the open promoter complexes for $u h p T$ transcription and also enhances the rate of their formation [49].

(ii) Modification of the antibiotic target MurA. Modification of the antibiotic target is one of the most common mechanisms to acquire antibiotic resistance in bacteria. MurA, an essential enzyme, is the target of the antibiotic fosfomycin, which, as mentioned, inactivates the enzyme by irreversibly binding to the protein. In E. coli mutation of the fosfomycin-binding site in MurA, Cys115, results in resistance to this antibiotic [50]. 
After a protein mutagenesis analysis, it has been proposed that the catalytic residue, Cys115, acts as a general acid-base catalyst, promoting the enzymatic reaction. When the enzyme mediates the enolpyruvyl transfer from PEP to the 3'-OH of UDP-GlcNAc, MurA-Cys115 reacts with PEP (or fosfomycin) to form a covalent phospholactoyl-enzyme adduct [15,51-53]. In addition, MurA-Cys115 seems to be essential for product release, i.e., inorganic phosphate and UDP-GlcNAc-3-O-enolpyruvate [54].

MurA shows an enzymatic activity susceptible to be blocked by fosfomycin in a dose-dependent manner. However, the Cys115 to Asp mutation in the E. coli MurA generates a fully active enzyme, yet completely insensitive to inhibition by fosfomycin, while the Cys115 to Glu mutant shows no enzymatic activity [50]. The impact of these types of mutations in the acquisition of fosfomycin resistance is reflected by the presence of an Asp residue in the catalytic site of MurA proteins encoded by pathogenic bacteria with intrinsic resistance to fosfomycin, such as Mycobacterium tuberculosis, Chlamydia trachomatis and Borrelia burgdorferi [55-57]. In addition, antibiotic resistance to fosfomycin was acquired in $E$. coli by the expression of this naturally resistant enzyme when endogenous E. coli murA was conditionally inactivated [57]. Conversely, mutation of the wild-type aspartate residue in the MurA active site to a cysteine renders an enzyme sensitive to fosfomycin in M. tuberculosis and B. burgdorferi $[55,56]$.

Modification of the fosfomycin target to acquire antibiotic resistance seems to be rare in clinical isolates. A fosfomycin-resistant E. coli mutant affected in MurA affinity to the antibiotic was characterized [58]. However, very few reports of clinical isolates show mutations in the murA gene, and none in the catalytic site of MurA. Recently, mutations in the MurA sequence of clinical isolates of E. coli, Asp369 to Asn and Leu370 to Ile, have been suggested to contribute to the development of fosfomycin resistance in vivo [59]. These mutant proteins are less susceptible to the inhibitory activity of this antibiotic. Both highly conserved residues could be important for PEP substrate binding and, thus, may affect interaction between the enzyme and fosfomycin [59].

Since fosfomycin produces covalent modification of MurA, increased synthesis of the enzyme confers a resistant phenotype [60]. Indeed, the analysis of a complete E. coli library of gene amplifications (the ASKA collection) has revealed that murA is the only gene in the entire E. coli genome capable of conferring clinical levels of antibiotic resistance when overexpressed [61]. Increased MurA levels in E. coli correlate with higher levels of fosfomycin resistance, reaching clinical resistance levels $(32 \mu \mathrm{g} / \mathrm{mL})$ at a low fitness cost. In this sense, it has been shown that the enhanced expression of the murA gene contributes to the acquisition of fosfomycin resistance in several E. coli clinical isolates [62].

(iii) Antibiotic modification. Several enzymes are able to modify fosfomycin, producing chemical changes that inactivate it. Microbial resistance to fosfomycin by antibiotic modification in pathogenic strains involves one of three different fosfomycin resistance proteins (FosA, FosB or FosX). All of them catalyse the opening of the oxirane ring of the antibiotic, rendering it ineffective. Nevertheless, they differ in terms of chemical mechanism, using different substrates to add chemical groups to the antibiotic [63].

Structure-based sequence alignments of Fos proteins show remarkable sequence homology among them, with a limited set of residues that differ among Fos enzymes and confer different catalytic properties to each class (Figure 4). This allows different fos genes to recombine via homologous 
recombination, leading to recombinant enzymes that confer fosfomycin resistance, as shown between Mesorhizobium loti FosX and P. aeruginosa FosA [64].

Figure 4. Amino acid sequence alignment generated by ClustalX (under Bioedict) of three representative sequences of fosfomycin resistance proteins (Fos) present in different bacterial species. Represented sequences are FosA [P. aeruginosa 18A], FosA3 [E. coli], FosB [S. aureus subsp. aureus USA300_TCH1516], FosC2 [E. coli] and FosX [Clostridium botulinum $\mathrm{Ba} 4$ str. 657]. Fos enzymes belong to the same divalent metal-ion dependent metalloenzymes, the vicinal oxygen chelate superfamily (VOC), sharing a high number of core-conserved or identical residues in their sequences.

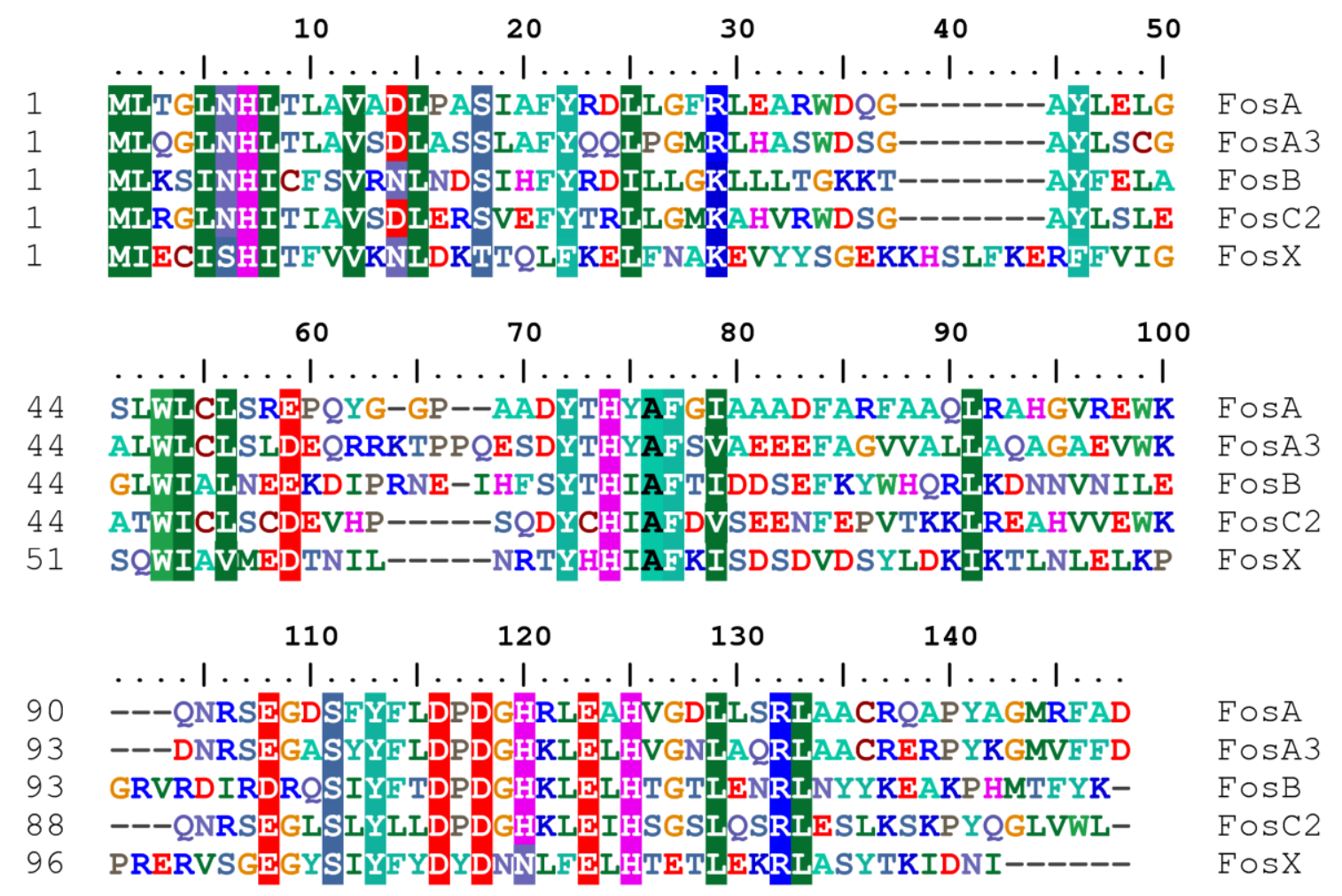

Fosfomycin resistance proteins (FosA, FosB and FosX) are members of the same metalloenzyme superfamily, the divalent metal-ion dependent enzymes [63]. They are evolutionarily related and form a group of enzymes related to glyoxalase I, methylmalonyl-CoA epimerase and extradiol dioxygenases, all members of the same metalloenzyme superfamily. The members of the metalloenzyme superfamily, the vicinal oxygen chelate superfamily (VOC), share a common structural fold that provides a very versatile metal coordination environment, mediating the catalysis of a very diverse set of reactions $[65,66]$.

FosA. FosA was first identified as a fosfomycin resistance determinant able to modify and inactivate the antibiotic in conjugative multiresistance plasmids from Enterobacteriaceae clinical isolates [67-69]. The gene fos $A$ was found to reside in a transposon, Tn2921, in some plasmids [70]. The nucleotide sequence of this transposon has revealed that $f o s A$ is flanked by two identical insertion sequences (ISs) and associated with genes showing striking similarity to a genomic segment from Enterobacter cancerogenus [71]. In fact, close relatives of FosA, with catalytic properties very similar to those of the plasmid-encoded enzyme, also appear in microbial genomes, such as that of the pathogen $P$. aeruginosa [72]. 
The fosfomycin resistance protein, FosA, is an $\mathrm{Mn}^{2+}$-dependent glutathione S-transferase that inactivates fosfomycin by the addition of glutathione to the oxirane ring of fosfomycin, rendering it inactive [73,74] (Figure 5). Overexpression of FosA from a plasmid in E. coli confers fosfomycin resistance in a wild-type strain, but not in cells deficient in glutathione biosynthesis [75]. FosA acts as a homodimeric metalloenzyme with an $\mathrm{Mn}^{2+}$ molecule bound to each subunit in a metal binding site that interacts strongly with the substrate fosfomycin $[72,75,76]$. In addition, FosA also requires $\mathrm{K}^{+}$for optimal activity, due to the 100-fold activation effect of the monovalent cation when it interacts with the catalytic site [77]. Functional analysis of the FosA sequence has revealed several residues involved in substrate binding and ligands to the $\mathrm{Mn}^{2+}$ and the $\mathrm{K}^{+}$ions that are essential for enzymatic activity [78].

Figure 5. Reactions catalysed by Fos metalloenzymes (FosA, FosB and FosX) and fosfomycin kinases (FosC, FomA and FomB). Fosfomycin-inactivating enzymes modify the antibiotic, rendering it inactive by opening the oxirane ring (metalloenzymes) or by phosphorylation (fosfomycin kinases). Substrates and the metal requirement for each enzyme are also shown.

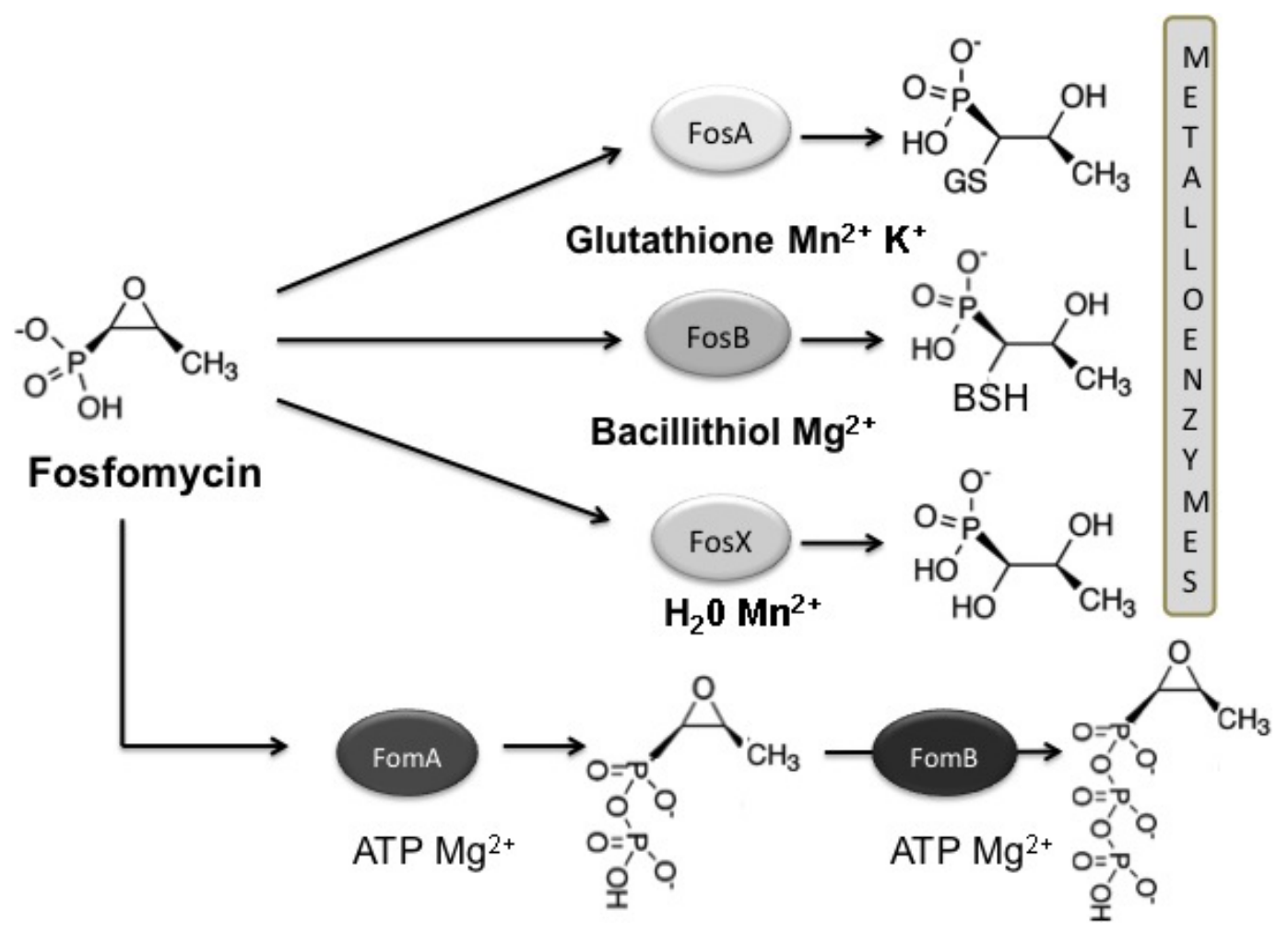

FosB. FosB is a thiol-S-transferase related to FosA that was first detected in a plasmid conferring resistance to fosfomycin in Staphylococcus epidermidis $[79,80]$. Fosb has been widely detected in the chromosomes and plasmids of many low G+C Gram-positive bacteria, including Bacillus subtilis, Bacillus anthracis, Bacillus cereus, S. aureus, S. epidermidis and E. faecium.

In $B$. subtilis, intrinsic fosfomycin resistance depends on the presence of the fos $B(y n d N)$ gene in the bacterial chromosome. Expression of $f \circ s B$ requires the extracytoplasmic sigma factor, SigW [81], a regulator with a prominent role in providing inducible resistance to antimicrobial compounds [82]. Therefore, fos $B$ or sig $W$ mutants are fosfomycin-sensitive in B. subtilis [81]. 
FosB was initially characterized as an $\mathrm{Mg}^{2+}$-dependent L-cysteine thiol transferase that catalyses the addition of a thiol group using L-cysteine as a donor substrate [81]. B. subtilis FosB is a dimer whose activity is almost 10 -fold greater with $\mathrm{Mg}^{2+}$ or $\mathrm{Ni}^{2+}$ than with $\mathrm{Mn}^{2+}$ as a cofactor. In contrast to FosA, FosB shows no monovalent metal dependence [81].

Recently, it has been suggested that bacillithiol, the $\alpha$-anomeric glycoside of L-cysteinyl-D-glucosamine with L-malic acid could be the thiol donor in vivo for FosB [83] (Figure 5). Cells lacking bacillithiol show a dramatic increase in fosfomycin sensitivity in B. subtilis, B. anthracis and S. aureus [83-85]. The increase in fosfomycin sensitivity, due to the lack of bacillithiol in $B$. subtilis, was similar to a $\operatorname{fos} B$ null mutant and to a $\operatorname{fos} B$ and bacillithiol biosynthesis double mutant [83]. Kinetic analysis of FosB from $S$. aureus has confirmed that the enzyme is a divalent metal-dependent $\left(\mathrm{Mg}^{2+}\right.$ and $\left.\mathrm{Mn}^{2+}\right)$ thiol S-transferase, and bacillithiol is its preferred thiol substrate under physiological conditions [85].

FosX. FosX hydrolases are a subfamily of enzymes related to FosA and FosB, sharing 30\%-35\% sequence identity with both groups of enzymes and detected in the survey of the microbial genome sequences [86]. fos $X$ homologues have been identified in the chromosome of several microorganisms, including Mesorhizobium loti, and the pathogens, L. monocytogenes, Clostrium botulinum and Brucella melitensis.

FosX is a $\mathrm{Mn}^{2+}$-dependent epoxide hydrolase that catalyses the hydration of fosfomycin [86,87] (Figure 5). The enzyme mediates the addition of water to the $\mathrm{C} 1$ position of the antibiotic, breaking the oxirane ring, producing a diol product. The reaction involves an essential glutamic acid residue in the Fos X active site acting as a general base catalyst for the reaction [86,87].

Other fosfomycin-inactivating enzymes. Microorganisms able to synthesize fosfomycin, such as some strains of Streptomyces and Pseudomonas syringae, are resistant to high concentrations of the antibiotic. Antibiotic-producing organisms usually associate biosynthetic genes to resistance genes in gene clusters in order to protect cells from the harmful effect of the antibiotic $[88,89]$.

Fosfomycin producers have antibiotic kinases unrelated to Fos metalloenzymes that modify and detoxify the antibiotic inside the cells. In Streptomyces spp., two fosfomycin kinases sequentially modify the antibiotic in the presence of ATP and $\mathrm{Mg}^{2+}$. FomA converts fosfomycin to fosfomycin monophosphate, while FomB produces fosfomycin diphosphate using the monophosphate form as a substrate [88,89] (Figure 5). FomA shares homology with the amino acid kinase family and also with the resistance protein, FosC, from $P$. syringae and differs from FomB. The structure of the FomA enzyme bound to fosfomycin shows a typical fold of the amino acid kinase family of enzymes, and important structural similarities [90]. Intrinsic resistance to fosfomycin in P. syringae relies on a fosfomycin kinase named $\mathrm{FosC}$, which is able to phosphorylate the antibiotic to produce fosfomycin monophosphate using ATP in the reaction [91] (Figure 5). However, a recent article demonstrated that FosC from P. syringae is actually an ortholog of FomA [92].

\section{Clinical Impact}

In clinical isolates, the main mechanism for the development of fosfomycin resistance is a reduced permeability of the cell membrane. The contribution of plasmid-encoded inactivating enzymes to the overall resistance to fosfomycin in clinical strains seems to be low $[93,94]$. Modification of the 
antibiotic target, MurA, also seems to be a very rare mechanism in fosfomycin-resistant clinical isolates, although it is clearly very important for intrinsic resistance in some pathogens [55-57]. Only a few reports have described mutations in the murA sequence or murA expression that could be associated with fosfomycin resistance [59,62]. This suggests that changes in the MurA sequence reducing its affinity for fosfomycin can also affect the essential process of peptidoglycan biosynthesis. This could be explained by the fact that MurA is an essential enzyme and, apparently, can be modified by point mutations in a few specific residues without rendering it inactive. In our experience, the probability of finding a murA mutant resistant to fosfomycin in a bacterial population is around $10^{-9}$ to $10^{-10}$ in vitro, while gene inactivation ( $g l p T$, for instance) is three orders of magnitude higher.

An analysis of plasmid-encoded fosfomycin resistance in pathogenic bacteria has revealed a relatively low percentage of $\operatorname{fos} A$ and $\operatorname{fos} B$ genes among fosfomycin-resistant strains. Only five strains out of 219 fosfomycin-resistant isolates carried plasmids harbouring fos $A$ (three Enterobacteria) and fos $B$ (two staphylococci). The mechanism of antibiotic resistance in the other isolates was caused by the alteration of the chromosomally encoded antibiotic uptake systems [93]. No transferable plasmid-encoded fosfomycin resistance was found among a collection of $P$. aeruginosa antibiotic resistance strains [95].

Although the incidence of plasmid-encoded fosfomycin-modifying enzymes is still low, plasmids that encode Fos enzymes are very often associated with other antibiotic resistance genes, leading to the emergence of multidrug resistance strains. In a survey of CTX-M $\beta$-lactamase-producing E. coli clinical isolates, three out of ten fosfomycin-resistant strains contained two different FosA-like enzymes with glutathione S-transferase activity, FosA3 and FosC2, encoded in transferable multiresistance plasmids simultaneously conferring a cefotaxime resistance phenotype [96]. Multiresistance plasmids encoding fos $A 3$ and, to a lesser extent, fos $C 2$ are emerging among CTX-M $\beta$-lactamase-producing E. coli and K. pneumonia isolates in Asia (China, Japan and South Korea) [96-99]. For instance, the multidrug resistance plasmid, pHN7A8, carries the bla(TEM-1b), bla(CTX-M-65), fosA3 and rmtB genes conferring resistance to penicillins, cephalosporins, fosfomycin and aminoglycosides, respectively [100]. Plasmid pKP96 carries nine genes (fos $A$ among them), conferring resistance to several antibiotics, including penicillins, cephalosporins, fosfomycin, aminoglycosides, tetracycline, quinolones and sulfamethoxazole [101]. Therefore, the acquisition of fosfomycin resistance mediated by antibioticmodifying enzymes shows a higher incidence in multidrug resistance strains. From a collection of 21 isolates with extended-spectrum $\beta$-lactamase, seven strains (five E. coli and two K. pneumoniae) harboured both fos $A 3$ and blaCTX connected via insertion sequences in different multiresistance plasmids [102]. Fos enzymes encoded in the chromosome contribute to intrinsic resistance, but they could also be important in pathogenic bacteria for the development of antibiotic resistance, as seen in $P$. aeruginosa overexpressing chromosomally encoded fos $A$ [103].

A recent study of six fosfomycin-resistant $E$. coli clinical isolates showed that all of them contained glpT mutations with an impaired GlpT transport system, and five of them were unable to grow using glycerol-3-P. Two of these strains with a high level of fosfomycin resistance were also defective in UhpT by gene loss, but all the other resistant isolates were functional in UhpT transport growing with glucose-6-P [59]. A previous report by Nilsson et al [104] about the molecular mechanism of fosfomycin resistance in $13 \mathrm{E}$. coli clinical isolates revealed that the highest level of fosfomycin resistance required simultaneous inactivation of both transport pathways, GlpT and UhpT (a glpT stop codon $+u h p A$ deletion in this strain). By contrast, most of the resistant strains analysed were only defective in the 
UhpT transport system, growing with glycerol-3-P, but not with glucose-6-P as a sole carbon source. Nevertheless, among those strains, the $u h p T$ and/or $u h p A$ genes, inactivating mutations were only detected in four of them, although it is possible missense mutations were not evaluated [104].

In a survey of fosfomycin-resistant isolates in the urinary isolates of $E$. coli producing extended-spectrum beta-lactamases, a cluster of five isolates carried an $u h p A$ deletion [105], showing that mutations targeted to chromosome genes could be important for the development of antibiotic multiresistance. Although mutations in $u h p A$ are often detected in clinical isolates, this is not the case for $u h p B$ or $u h p C$. Experiments of in vitro mutagenesis by insertions in $u h p A$ led to the loss of $u h p T$ expression; however, a high proportion of $u p h B$ or $u h p C$ mutations retained $u h p T$ expression [39]. A high level of fosfomycin resistance has also been described by the concurrent effects of increased murA expression/murA point mutations and alteration of the GlpT or/and UhpT transport systems in two Shiga-Like Toxin-producing E. coli strains [59,62].

Finally, it is known that mutations in cyaA or ptsI, which in turn decrease the level of cAMP, provoke a profound disturbance in carbohydrate metabolism of bacteria and may have a high biological cost [104]. Therefore, it is expected that these mutations per se lack clinical relevance unless bacteria find compensatory mutations. The need for this compensation certainly diminishes the probability of finding these mutants in clinical settings.

The sequence changes in fosfomycin-resistant strains isolated both in vitro and in clinical settings show that large and small deletions are the main source of gene-inactivating mutations, followed by insertions/duplications. In addition, a considerable number of point mutations have been detected, including truncations by nonsense and missense mutations $[20,104,106]$. As an example, the sequence of $g l p T$ in 20 fosfomycin-resistant isolates of $P$. aeruginosa, where all fosfomycin resistance mutations are targeted to $g l p T$, has been explored detecting 14 deletions (nine frameshifts), five-point mutations and one insertion/duplication [20,106].

\section{Conclusions}

Fosfomycin has been used for a long time, but the emergence of antibiotic resistance and the decline in newly developed antibiotics has increased interest in the treatment of bacterial infections with this antibiotic. The mechanisms of acquisition of fosfomycin resistance should be considered in detail so as to optimize therapy and avoid the further development of antibiotic resistance. Evaluation of fosfomycin susceptibility in clinical strains is widely performed, but the molecular bases are frequently unexplored. In addition, several clinical trials have recently been performed to evaluate the potential application of fosfomycin in the treatment of bacterial infections, including those caused by multidrug resistant isolates, most of them with promising results. Therefore, a more-in-depth knowledge of the molecular mechanisms leading to fosfomycin resistance in clinical strains could improve the successful use of fosfomycin for the treatment of bacterial infections.

\section{Acknowledgements}

This work was supported by the Ministerio de Economía y Competitividad, Instituto de Salud Carlos III, co-financed by the European Development Regional Fund "A way to achieve Europe" ERDF, Spanish Network for the Research in Infectious Diseases (REIPI RD12/0015 and FIS 
PI10/00105) and the PAR Project (Ref 241476) of the EU 7th Framework Programme. ACG was supported by a postdoctoral grant in life sciences from the Fundacion Ramon Areces. ARR was supported by The Collaborative Research Centre (CRC) 973 "Priming and Memory of Organismic Responses to Stress". Some of the figures were generated from the online pathways chart of SABioscience.

\section{Conflict of Interest}

The authors declare no conflict of interest.

\section{References and Notes}

1. Nathan, C. Antibiotics at the crossroads. Nature 2004, 431, 899-902.

2. Livermore, D.M. Has the era of untreatable infections arrived? J. Antimicrob. Chemother. 2009, 64 (Suppl. 1), 29-36.

3. Falagas, M.E.; Grammatikos, A.P.; Michalopoulos, A. Potential of old-generation antibiotics to address current need for new antibiotics. Expert Rev. Anti. Infect. Ther. 2008, 6, 593-600.

4. Hendlin, D.; Stapley, E.O.; Jackson, M.; Wallick, H.; Miller, A.K.; Wolf, F.J.; Miller, T.W.; Chaiet, L.; Kahan, F.M.; Foltz, E.L.; et al. Phosphonomycin, a new antibiotic produced by strains of Streptomyces. Science 1969, 166, 122-123.

5. Kahan, F.M.; Kahan, J.S.; Cassidy, P.J.; Kropp, H. The mechanism of action of fosfomycin (phosphonomycin). Ann. NY Acad. Sci. 1974, 235, 364-386.

6. Wagenlehner, F.M.; Hoyme, U.; Kaase, M.; Funfstuck, R.; Naber, K.G.; Schmiemann, G. Uncomplicated urinary tract infections. Dtsch. Arztebl. Int. 2011, 108, 415-423.

7. Schito, G.C. Why fosfomycin trometamol as first line therapy for uncomplicated UTI? Int. J. Antimicrob. Agents 2003, 22 (Suppl. 2), 79-83.

8. Falagas, M.E.; Giannopoulou, K.P.; Kokolakis, G.N.; Rafailidis, P.I. Fosfomycin: Use beyond urinary tract and gastrointestinal infections. Clin. Infect. Dis. 2008, 46, 1069-1077.

9. Barry, A.L.; Brown, S.D. Antibacterial spectrum of fosfomycin trometamol. J. Antimicrob. Chemother. 1995, 35, 228-230.

10. Lu, C.L.; Liu, C.Y.; Huang, Y.T.; Liao, C.H.; Teng, L.J.; Turnidge, J.D.; Hsueh, P.R. Antimicrobial susceptibilities of commonly encountered bacterial isolates to fosfomycin determined by agar dilution and disk diffusion methods. Antimicrob. Agents Chemother. 2011, $55,4295-4301$.

11. Falagas, M.E.; Kastoris, A.C.; Karageorgopoulos, D.E.; Rafailidis, P.I. Fosfomycin for the treatment of infections caused by multidrug-resistant non-fermenting gram-negative bacilli: A systematic review of microbiological, animal and clinical studies. Int. J. Antimicrob. Agents 2009, 34, 111-120.

12. Neuner, E.A.; Sekeres, J.; Hall, G.S.; van Duin, D. Experience with fosfomycin for treatment of urinary tract infections due to multidrug-resistant organisms. Antimicrob. Agents Chemother. 2012, 56, 5744-5748.

13. Falagas, M.E.; Kastoris, A.C.; Kapaskelis, A.M.; Karageorgopoulos, D.E. Fosfomycin for the treatment of multidrug-resistant, including extended-spectrum beta-lactamase producing, Enterobacteriaceae infections: A systematic review. Lancet Infect. Dis. 2010, 10, 43-50. 
14. Brown, E.D.; Vivas, E.I.; Walsh, C.T.; Kolter, R. MurA (MurZ), the enzyme that catalyzes the first committed step in peptidoglycan biosynthesis, is essential in Escherichia coli. J. Bacteriol. 1995, 177, 4194-4197.

15. Marquardt, J.L.; Brown, E.D.; Lane, W.S.; Haley, T.M.; Ichikawa, Y.; Wong, C.H.; Walsh, C.T. Kinetics, stoichiometry, and identification of the reactive thiolate in the inactivation of UDP-GlcNAc enolpyruvoyl transferase by the antibiotic fosfomycin. Biochemistry 1994, 33, 10646-10651.

16. Skarzynski, T.; Mistry, A.; Wonacott, A.; Hutchinson, S.E.; Kelly, V.A.; Duncan, K. Structure of UDP-N-acetylglucosamine enolpyruvyl transferase, an enzyme essential for the synthesis of bacterial peptidoglycan, complexed with substrate UDP-N-acetylglucosamine and the drug fosfomycin. Structure 1996, 4, 1465-1474.

17. Kadner, R.J.; Winkler, H.H. Isolation and characterization of mutations affecting the transport of hexose phosphates in Escherichia coli. J. Bacteriol. 1973, 113, 895-900.

18. Tsuruoka, T.; Yamada, Y. Charactertization of spontaneous fosfomycin (phosphonomycin)-resistant cells of Escherichia coli B in vitro. J. Antibiot. (Tokyo) 1975, 28, 906-911.

19. Grimm, H. In vitro investigations with fosfomycin on Mueller-Hinton agar with and without glucose-6-phosphate. Infection 1979, 7, 256-259.

20. Castaneda-Garcia, A.; Rodriguez-Rojas, A.; Guelfo, J.R.; Blazquez, J. The glycerol-3-phosphate permease GlpT is the only fosfomycin transporter in Pseudomonas aeruginosa. J. Bacteriol. 2009, 191, 6968-6974.

21. Schweizer, H.P.; Po, C. Regulation of glycerol metabolism in Pseudomonas aeruginosa: Characterization of the glpR repressor gene. J. Bacteriol. 1996, 178, 5215-5221.

22. Scortti, M.; Lacharme-Lora, L.; Wagner, M.; Chico-Calero, I.; Losito, P.; Vazquez-Boland, J.A. Coexpression of virulence and fosfomycin susceptibility in Listeria: Molecular basis of an antimicrobial in vitro-in vivo paradox. Nat. Med. 2006, 12, 515-517.

23. Chico-Calero, I.; Suarez, M.; Gonzalez-Zorn, B.; Scortti, M.; Slaghuis, J.; Goebel, W.; Vazquez-Boland, J.A. Hpt, a bacterial homolog of the microsomal glucose-6-phosphate translocase, mediates rapid intracellular proliferation in Listeria. Proc. Natl. Acad. Sci. USA 2002, 99, 431-436.

24. Lemieux, M.J.; Huang, Y.; Wang, D.N. Glycerol-3-phosphate transporter of Escherichia coli: Structure, function and regulation. Res. Microbiol. 2004, 155, 623-629.

25. Huang, Y.; Lemieux, M.J.; Song, J.; Auer, M.; Wang, D.N. Structure and mechanism of the glycerol-3-phosphate transporter from Escherichia coli. Science 2003, 301, 616-620.

26. Lemieux, M.J.; Huang, Y.; Wang, D.N. The structural basis of substrate translocation by the Escherichia coli glycerol-3-phosphate transporter: A member of the major facilitator superfamily. Curr. Opin. Struct. Biol. 2004, 14, 405-412.

27. Elvin, C.M.; Hardy, C.M.; Rosenberg, H. Pi exchange mediated by the glpt-dependent sn-glycerol-3-phosphate transport system in Escherichia coli. J. Bacteriol. 1985, 161, 1054-1058.

28. Hardisson, C.; Llaneza, J. The action of fosfomycin on the growth of Pseudomonas aeruginosa. Chemotherapy 1977, 23 (Suppl. 1), 37-44.

29. Lindgren, V. Mapping of a genetic locus that affects glycerol 3-phosphate transport in Bacillus subtilis. J. Bacteriol. 1978, 133, 667-670. 
30. Santoro, A.; Cappello, A.R.; Madeo, M.; Martello, E.; Iacopetta, D.; Dolce, V. Interaction of fosfomycin with the glycerol 3-phosphate transporter of Escherichia coli. Biochim. Biophys. Acta 2011, 1810, 1323-1329.

31. Larson, T.J.; Ye, S.Z.; Weissenborn, D.L.; Hoffmann, H.J.; Schweizer, H. Purification and characterization of the repressor for the sn-glycerol 3-phosphate regulon of Escherichia coli K-12. J. Biol. Chem. 1987, 262, 15869-15874.

32. Yang, B.; Gerhardt, S.G.; Larson, T.J. Action at a distance for $g l p$ repressor control of $g l p T Q$ transcription in Escherichia coli K-12. Mol. Microbiol. 1997, 24, 511-521.

33. Zeng, G.; Ye, S.; Larson, T.J. Repressor for the sn-glycerol 3-phosphate regulon of Escherichia coli K-12: Primary structure and identification of the DNA-binding domain. J. Bacteriol. 1996, 178, 7080-7089.

34. Kadner, R.J.; Shattuck-Eidens, D.M. Genetic control of the hexose phosphate transport system of Escherichia coli: Mapping of deletion and insertion mutations in the uhp region. J. Bacteriol. 1983, 155, 1052-1061.

35. Sonna, L.A.; Ambudkar, S.V.; Maloney, P.C. The mechanism of glucose 6-phosphate transport by Escherichia coli. J. Biol. Chem. 1988, 263, 6625-6630.

36. Eiglmeier, K.; Boos, W.; Cole, S.T. Nucleotide sequence and transcriptional startpoint of the glpT gene of Escherichia coli: Extensive sequence homology of the glycerol-3-phosphate transport protein with components of the hexose-6-phosphate transport system. Mol. Microbiol. 1987, 1, 251-258.

37. Ambudkar, S.V.; Anantharam, V.; Maloney, P.C. UhpT, the sugar phosphate antiporter of Escherichia coli, functions as a monomer. J. Biol. Chem. 1990, 265, 12287-12292.

38. Lloyd, A.D.; Kadner, R.J. Topology of the Escherichia coli uhpT sugar-phosphate transporter analyzed by using TnphoA fusions. J. Bacteriol. 1990, 172, 1688-1693.

39. Island, M.D.; Kadner, R.J. Interplay between the membrane-associated UhpB and UhpC regulatory proteins. J. Bacteriol. 1993, 175, 5028-5034.

40. Chen, Q.; Kadner, R.J. Effect of altered spacing between uhpT promoter elements on transcription activation. J. Bacteriol. 2000, 182, 4430-4436.

41. Dahl, J.L.; Wei, B.Y.; Kadner, R.J. Protein phosphorylation affects binding of the Escherichia coli transcription activator UhpA to the uhpT promoter. J. Biol. Chem. 1997, 272, 1910-1919.

42. Olekhnovich, I.N.; Kadner, R.J. Mutational scanning and affinity cleavage analysis of UhpA-binding sites in the Escherichia coli uhpT promoter. J. Bacteriol. 2002, 184, 2682-2691.

43. Alper, M.D.; Ames, B.N. Transport of antibiotics and metabolite analogs by systems under cyclic AMP control: Positive selection of Salmonella typhimurium cya and crp mutants. J. Bacteriol. 1978, 133, 149-157.

44. Cordaro, J.C.; Melton, T.; Stratis, J.P.; Atagun, M.; Gladding, C.; Hartman, P.E.; Roseman, S. Fosfomycin resistance: Selection method for internal and extended deletions of the phosphoenolpyruvate: Sugar phosphotransferase genes of Salmonella typhimurium. J. Bacteriol. 1976, 128, 785-793.

45. Sakamoto, Y.; Furukawa, S.; Ogihara, H.; Yamasaki, M. Fosmidomycin resistance in adenylate cyclase deficient (cya) mutants of Escherichia coli. Biosci. Biotechnol. Biochem. 2003, 67, 2030-2033. 
46. Tsuruoka, T.; Miyata, A.; Yamada, Y. Two kinds of mutants defective in multiple carbohydrate utilization isolated from in vitro fosfomycin-resistant strains of Escherichia coli K-12. J. Antibiot. (Tokyo) 1978, 31, 192-201.

47. Larson, T.J.; Cantwell, J.S.; van Loo-Bhattacharya, A.T. Interaction at a distance between multiple operators controls the adjacent, divergently transcribed $g l p T Q$-glpACB operons of Escherichia coli K-12. J. Biol. Chem. 1992, 267, 6114-6121.

48. Merkel, T.J.; Dahl, J.L.; Ebright, R.H.; Kadner, R.J. Transcription activation at the Escherichia coli uhpT promoter by the catabolite gene activator protein. J. Bacteriol. 1995, 177, 1712-1718.

49. Olekhnovich, I.N.; Dahl, J.L.; Kadner, R.J. Separate contributions of UhpA and CAP to activation of transcription of the $u h p T$ promoter of Escherichia coli. J. Mol. Biol. 1999, 292, 973-986.

50. Kim, D.H.; Lees, W.J.; Kempsell, K.E.; Lane, W.S.; Duncan, K.; Walsh, C.T. Characterization of a Cys115 to Asp substitution in the Escherichia coli cell wall biosynthetic enzyme UDP-GlcNAc enolpyruvyl transferase (MurA) that confers resistance to inactivation by the antibiotic fosfomycin. Biochemistry 1996, 35, 4923-4928.

51. Brown, E.D.; Marquardt, J.L.; Lee, J.P.; Walsh, C.T.; Anderson, K.S. Detection and characterization of a phospholactoyl-enzyme adduct in the reaction catalyzed by UDP-N-acetylglucosamine enolpyruvoyl transferase, MurZ. Biochemistry 1994, 33, 10638-10645.

52. Ramilo, C.; Appleyard, R.J.; Wanke, C.; Krekel, F.; Amrhein, N.; Evans, J.N. Detection of the covalent intermediate of UDP-N-acetylglucosamine enolpyruvyl transferase by solution-state and time-resolved solid-state NMR spectroscopy. Biochemistry 1994, 33, 15071-15079.

53. Wanke, C.; Amrhein, N. Evidence that the reaction of the UDP-N-acetylglucosamine 1-carboxyvinyltransferase proceeds through the O-phosphothioketal of pyruvic acid bound to Cys 115 of the enzyme. Eur. J. Biochem. 1993, 218, 861-870.

54. Eschenburg, S.; Priestman, M.; Schonbrunn, E. Evidence that the fosfomycin target Cys115 in UDP-N-acetylglucosamine enolpyruvyl transferase (MurA) is essential for product release. J. Biol. Chem. 2005, 280, 3757-3763.

55. De Smet, K.A.; Kempsell, K.E.; Gallagher, A.; Duncan, K.; Young, D.B. Alteration of a single amino acid residue reverses fosfomycin resistance of recombinant MurA from Mycobacterium tuberculosis. Microbiology 1999, 145, 3177-3184.

56. Jiang, S.; Gilpin, M.E.; Attia, M.; Ting, Y.L.; Berti, P.J. Lyme disease enolpyruvyl-udp-glcnac synthase: Fosfomycin-resistant MurA from Borrelia burgdorferi, a fosfomycin-sensitive mutant, and the catalytic role of the active site Asp. Biochemistry 2011, 50, 2205-2212.

57. McCoy, A.J.; Sandlin, R.C.; Maurelli, A.T. In vitro and in vivo functional activity of Chlamydia MurA, a UDP-N-acetylglucosamine enolpyruvyl transferase involved in peptidoglycan synthesis and fosfomycin resistance. J. Bacteriol. 2003, 185, 1218-1228.

58. Venkateswaran, P.S.; Wu, H.C. Isolation and characterization of a phosphonomycin-resistant mutant of Escherichia coli K-12. J. Bacteriol. 1972, 110, 935-944.

59. Takahata, S.; Ida, T.; Hiraishi, T.; Sakakibara, S.; Maebashi, K.; Terada, S.; Muratani, T.; Matsumoto, T.; Nakahama, C.; Tomono, K. Molecular mechanisms of fosfomycin resistance in clinical isolates of Escherichia coli. Int. J. Antimicrob. Agents 2010, 35, 333-337. 
60. Marquardt, J.L.; Siegele, D.A.; Kolter, R.; Walsh, C.T. Cloning and sequencing of Escherichia coli MurZ and purification of its product, a UDP-N-acetylglucosamine enolpyruvyl transferase. J. Bacteriol. 1992, 174, 5748-5752.

61. Couce, A.; Briales, A.; Rodriguez-Rojas, A.; Costas, C.; Pascual, A.; Blazquez, J. Genomewide overexpression screen for fosfomycin resistance in Escherichia coli: MurA confers clinical resistance at low fitness cost. Antimicrob. Agents Chemother. 2012, 56, 2767-2769.

62. Horii, T.; Kimura, T.; Sato, K.; Shibayama, K.; Ohta, M. Emergence of fosfomycin-resistant isolates of shiga-like toxin-producing Escherichia coli O26. Antimicrob. Agents Chemother. 1999, 43, 789-793.

63. Rigsby, R.E.; Fillgrove, K.L.; Beihoffer, L.A.; Armstrong, R.N. Fosfomycin resistance proteins: A nexus of glutathione transferases and epoxide hydrolases in a metalloenzyme superfamily. Meth. Enzymol. 2005, 401, 367-379.

64. Brown, D.W.; Schaab, M.R.; Birmingham, W.R.; Armstrong, R.N. Evolution of the antibiotic resistance protein, FosA, is linked to a catalytically promiscuous progenitor. Biochemistry 2009, 48, 1847-1849.

65. Armstrong, R.N. Mechanistic imperatives for the evolution of glutathione transferases. Curr. Opin. Chem. Biol. 1998, 2, 618-623.

66. Armstrong, R.N. Mechanistic diversity in a metalloenzyme superfamily. Biochemistry 2000, 39, 13625-13632.

67. Leon, J.; Garcia-Lobo, J.M.; Navas, J.; Ortiz, J.M. Fosfomycin-resistance plasmids determine an intracellular modification of fosfomycin. J. Gen. Microbiol. 1985, 131, 1649-1655.

68. Llaneza, J.; Villar, C.J.; Salas, J.A.; Suarez, J.E.; Mendoza, M.C.; Hardisson, C. Plasmid-mediated fosfomycin resistance is due to enzymatic modification of the antibiotic. Antimicrob. Agents Chemother. 1985, 28, 163-164.

69. Mendoza, C.; Garcia, J.M.; Llaneza, J.; Mendez, F.J.; Hardisson, C.; Ortiz, J.M. Plasmid-determined resistance to fosfomycin in Serratia marcescens. Antimicrob. Agents Chemother. 1980, 18, 215-219.

70. Garcia-Lobo, J.M.; Ortiz, J.M. Tn2921, a transposon encoding fosfomycin resistance. J. Bacteriol. 1982, 151, 477-479.

71. Seoane, A.; Sangari, F.J.; Lobo, J.M. Complete nucleotide sequence of the fosfomycin resistance transposon Tn2921. Int. J. Antimicrob. Agents 2010, 35, 413-414.

72. Rife, C.L.; Pharris, R.E.; Newcomer, M.E.; Armstrong, R.N. Crystal structure of a genomically encoded fosfomycin resistance protein (FosA) at 1.19 A resolution by MAD phasing off the L-III edge of Tl(+). J. Am. Chem. Soc. 2002, 124, 11001-11003.

73. Arca, P.; Hardisson, C.; Suarez, J.E. Purification of a glutathione S-transferase that mediates fosfomycin resistance in bacteria. Antimicrob. Agents Chemother. 1990, 34, 844-848.

74. Arca, P.; Rico, M.; Brana, A.F.; Villar, C.J.; Hardisson, C.; Suarez, J.E. Formation of an adduct between fosfomycin and glutathione: A new mechanism of antibiotic resistance in bacteria. Antimicrob. Agents Chemother. 1988, 32, 1552-1556.

75. Bernat, B.A.; Laughlin, L.T.; Armstrong, R.N. Fosfomycin resistance protein (FosA) is a manganese metalloglutathione transferase related to glyoxalase I and the extradiol dioxygenases. Biochemistry 1997, 36, 3050-3055. 
76. Bernat, B.A.; Armstrong, R.N. Elementary steps in the acquisition of $\mathrm{Mn}^{2+}$ by the fosfomycin resistance protein (FosA). Biochemistry 2001, 40, 12712-12718.

77. Bernat, B.A.; Laughlin, L.T.; Armstrong, R.N. Elucidation of a monovalent cation dependence and characterization of the divalent cation binding site of the fosfomycin resistance protein (FosA). Biochemistry 1999, 38, 7462-7469.

78. Beharry, Z.; Palzkill, T. Functional analysis of active site residues of the fosfomycin resistance enzyme FosA from Pseudomonas aeruginosa. J. Biol. Chem. 2005, 280, 17786-17791.

79. Etienne, J.; Gerbaud, G.; Fleurette, J.; Courvalin, P. Characterization of staphylococcal plasmids hybridizing with the fosfomycin resistance gene fosB. FEMS Microbiol. Lett. 1991, 68, 119-122.

80. Zilhao, R.; Courvalin, P. Nucleotide sequence of the $f o s B$ gene conferring fosfomycin resistance in Staphylococcus epidermidis. FEMS Microbiol. Lett. 1990, 56, 267-272.

81. Cao, M.; Bernat, B.A.; Wang, Z.; Armstrong, R.N.; Helmann, J.D. FosB, a cysteine-dependent fosfomycin resistance protein under the control of sigma(W), an extracytoplasmic-function sigma factor in Bacillus subtilis. J. Bacteriol. 2001, 183, 2380-2383.

82. Butcher, B.G.; Helmann, J.D. Identification of Bacillus subtilis sigma-dependent genes that provide intrinsic resistance to antimicrobial compounds produced by Bacilli. Mol. Microbiol. 2006, 60, 765-782.

83. Gaballa, A.; Newton, G.L.; Antelmann, H.; Parsonage, D.; Upton, H.; Rawat, M.; Claiborne, A.; Fahey, R.C.; Helmann, J.D. Biosynthesis and functions of bacillithiol, a major low-molecular-weight thiol in Bacilli. Proc. Natl. Acad. Sci. USA 2010, 107, 6482-6486.

84. Parsonage, D.; Newton, G.L.; Holder, R.C.; Wallace, B.D.; Paige, C.; Hamilton, C.J.; Dos Santos, P.C.; Redinbo, M.R.; Reid, S.D.; Claiborne, A. Characterization of the $\mathrm{N}$-acetyl-alpha-D-glucosaminyl 1-malate synthase and deacetylase functions for bacillithiol biosynthesis in Bacillus anthracis. Biochemistry 2010, 49, 8398-8414.

85. Roberts, A.A.; Sharma, S.V.; Strankman, A.W.; Duran, S.R.; Rawat, M.; Hamilton, C.J. Mechanistic studies of FosB: A divalent metal-dependent bacillithiol-S-transferase that mediates fosfomycin resistance in Staphylococcus aureus. Biochem. J. 2013, 451, 69-79.

86. Fillgrove, K.L.; Pakhomova, S.; Newcomer, M.E.; Armstrong, R.N. Mechanistic diversity of fosfomycin resistance in pathogenic microorganisms. J. Am. Chem. Soc. 2003, 125, 15730-15731.

87. Fillgrove, K.L.; Pakhomova, S.; Schaab, M.R.; Newcomer, M.E.; Armstrong, R.N. Structure and mechanism of the genomically encoded fosfomycin resistance protein, FosX, from Listeria monocytogenes. Biochemistry 2007, 46, 8110-8120.

88. Kobayashi, S.; Kuzuyama, T.; Seto, H. Characterization of the fomA and fomB gene products from Streptomyces wedmorensis, which confer fosfomycin resistance on Escherichia coli. Antimicrob. Agents Chemother. 2000, 44, 647-650.

89. Kuzuyama, T.; Kobayashi, S.; O'Hara, K.; Hidaka, T.; Seto, H. Fosfomycin monophosphate and fosfomycin diphosphate, two inactivated fosfomycin derivates formed by gene products of fom $A$ and $f_{0 m B}$ from a fosfomycin producing organism Streptomyces wedmorensis. J. Antibiot. (Tokyo) 1996, 49, 502-504.

90. Pakhomova, S.; Bartlett, S.G.; Augustus, A.; Kuzuyama, T.; Newcomer, M.E. Crystal structure of fosfomycin resistance kinase FomA from Streptomyces wedmorensis. J. Biol. Chem. 2008, 283, 28518-28526. 
91. Garcia, P.; Arca, P.; Evaristo Suarez, J. Product of fosC, a gene from Pseudomonas syringae, mediates fosfomycin resistance by using ATP as cosubstrate. Antimicrob. Agents Chemother. 1995, 39, 1569-1573.

92. Kim, S.Y.; Ju, K.S.; Metcalf, W.W.; Evans, B.S.; Kuzuyama, T.; van der Donk, W.A. Different biosynthetic pathways to fosfomycin in Pseudomonas syringae and Streptomyces species. Antimicrob. Agents Chemother. 2012, 56, 4175-4183.

93. Arca, P.; Reguera, G.; Hardisson, C. Plasmid-encoded fosfomycin resistance in bacteria isolated from the urinary tract in a multicentre survey. J. Antimicrob. Chemother. 1997, 40, 393-399.

94. O'Hara, K. Two different types of fosfomycin resistance in clinical isolates of Klebsiella pneumoniae. FEMS Microbiol. Lett. 1993, 114, 9-16.

95. Shimizu, M.; Shigeobu, F.; Miyakozawa, I.; Nakamura, A.; Suzuki, M.; Mizukoshi, S.; O’Hara, K.; Sawai, T. Novel fosfomycin resistance of Pseudomonas aeruginosa clinical isolates recovered in Japan in 1996. Antimicrob. Agents Chemother. 2000, 44, 2007-2008.

96. Wachino, J.; Yamane, K.; Suzuki, S.; Kimura, K.; Arakawa, Y. Prevalence of fosfomycin resistance among CTX-M-producing Escherichia coli clinical isolates in Japan and identification of novel plasmid-mediated fosfomycin-modifying enzymes. Antimicrob. Agents Chemother. 2010, 54, 3061-3064.

97. Ho, P.L.; Chan, J.; Lo, W.U.; Law, P.Y.; Chow, K.H. Plasmid-mediated fosfomycin resistance in Escherichia coli isolated from pig. Vet. Microbiol. 2013, 162, 964-967.

98. Ho, P.L.; Chan, J.; Lo, W.U.; Law, P.Y.; Li, Z.; Lai, E.L.; Chow, K.H., Dissemination of plasmid-mediated fosfomycin resistance fos $A 3$ among multidrug-resistant Escherichia coli from livestock and other animals. J. Appl. Microbiol. 2013, 114, 695-702.

99. Hou, J.; Huang, X.; Deng, Y.; He, L.; Yang, T.; Zeng, Z.; Chen, Z.; Liu, J.H. Dissemination of the fosfomycin resistance gene fos 33 with CTX-M beta-lactamase genes and $r m t B$ carried on IncFII plasmids among Escherichia coli isolates from pets in China. Antimicrob. Agents Chemother. 2012, 56, 2135-2138.

100. He, L.; Partridge, S.R.; Yang, X.; Hou, J.; Deng, Y.; Yao, Q.; Zeng, Z.; Chen, Z.; Liu, J.H. Complete nucleotide sequence of pHN7A8, an F33:A-:B-type epidemic plasmid carrying blaCTX-M-65, fosA3 and $r m t B$ from China. J. Antimicrob. Chemother. 2013, 68, 46-50.

101. Shen, P.; Jiang, Y.; Zhou, Z.; Zhang, J.; Yu, Y.; Li, L. Complete nucleotide sequence of pKP96, a 67850 bp multiresistance plasmid encoding qnrA1, aac(6')-Ib-cr and blaCTX-M-24 from Klebsiella pneumonia. J. Antimicrob. Chemother. 2008, 62, 1252-1256.

102. Lee, S.Y.; Park, Y.J.; Yu, J.K.; Jung, S.; Kim, Y.; Jeong, S.H.; Arakawa, Y. Prevalence of acquired fosfomycin resistance among extended-spectrum beta-lactamase-producing Escherichia coli and Klebsiella pneumoniae clinical isolates in Korea and IS26-composite transposon surrounding fosA3. J. Antimicrob. Chemother. 2012, 60, 329-336.

103. De Groote, V.N.; Fauvart, M.; Kint, C.I.; Verstraeten, N.; Jans, A.; Cornelis, P.; Michiels, J. Pseudomonas aeruginosa fosfomycin resistance mechanisms affect non-inherited fluoroquinolone tolerance. J. Med. Microbiol. 2011, 60, 329-336.

104. Nilsson, A.I.; Berg, O.G.; Aspevall, O.; Kahlmeter, G.; Andersson, D.I. Biological costs and mechanisms of fosfomycin resistance in Escherichia coli. Antimicrob. Agents Chemother. 2003, 47, 2850-2858. 
105. Oteo, J.; Orden, B.; Bautista, V.; Cuevas, O.; Arroyo, M.; Martinez-Ruiz, R.; Perez-Vazquez, M.; Alcaraz, M.; Garcia-Cobos, S.; Campos, J. CTX-M-15-producing urinary Escherichia coli O25b-ST131-phylogroup B2 has acquired resistance to fosfomycin. J. Antimicrob. Chemother. 2009, 64, 712-717.

106. Rodriguez-Rojas, A.; Macia, M.D.; Couce, A.; Gomez, C.; Castaneda-Garcia, A.; Oliver, A.; Blazquez, J. Assesing the emergence of resistance: The absence of biological cost in vivo may compromise fosfomycin treatments for Pseudomonas aeruginosa infections. PLoS One 2010, 5, e10193.

(C) 2013 by the authors; licensee MDPI, Basel, Switzerland. This article is an open access article distributed under the terms and conditions of the Creative Commons Attribution license (http://creativecommons.org/licenses/by/3.0/). 